\title{
Effects on the Radiation Characteristics of Using a Corrugated Reflector With a Helical Antenna and an Electromagnetic Band-Gap Reflector With a Spiral Antenna
}

\author{
Hisamatsu Nakano, Fellow, IEEE, Kazuo Hitosugi, Naoki Tatsuzawa, Daisuke Togashi, \\ Hiroaki Mimaki, Member, IEEE, and Junji Yamauchi, Member, IEEE
}

\begin{abstract}
An axial-mode helical antenna backed by a perfect electric conductor (PEC reflector) is optimized to radiate a circularly polarized (CP) wave, using the finite-difference time-domain method (FDTDM). After the optimization, the PEC reflector is replaced with a corrugated reflector. The effects of the corrugated reflector on the current distribution along the helical arm and the radiation pattern are investigated. A reduction in the backward radiation is attributed to the reduction in the current flowing over the rear surface of the corrugated reflector. A spiral antenna backed by a PEC reflector of finite extent is also analyzed using the FDTDM. As the antenna height decreases, the reverse current toward the feed point increases, resulting in deterioration of the axial ratio. To overcome this deterioration, the $\mathrm{PEC}$ reflector is replaced with an electromagnetic band-gap (EBG) reflector composed of mushroom-like elements. Analysis reveals that the spiral radiates a $\mathrm{CP}$ wave even when the spiral is located close to the reflector $(0.06$ wavelength above the EBG surface). The input impedance for the EBG reflector is more stable over a wide frequency band than that for the PEC reflector.
\end{abstract}

Index Terms-Corrugated reflector, electromagnetic band-gap reflector, finite-difference time-domain method (FDTDM), helical antenna, spiral antenna.

\section{INTRODUCTION}

$\mathbf{T}$ HIS PAPER presents two antennas: one is a helical antenna and the other a spiral antenna. The basic radiation characteristics of these antennas have already been experimentally and theoretically investigated [1]-[4] and it has been revealed that the radiation characteristics from these antennas depend on their respective circumferences.

When a helical arm is wound on a virtual cylinder of a onewavelength circumference (for a given design frequency), the helix radiates a circularly polarized (CP) wave in the direction of the antenna axis. This is called axial-mode radiation [1]. Note that a perfect electric conductor (PEC) backs the helix to obtain such axial-mode radiation. Also, note that, as the size of the PEC plane (which acts as a reflector) decreases, the backward radiation increases [2].

The circumference of a spiral antenna must be more than one wavelength at the design frequency to radiate a $\mathrm{CP}$ wave. The

Manuscript received November 25, 2003; revised July 19, 2004.

The authors are with the College of Engineering, Hosei University, Koganei, Tokyo 184-8584, Japan (e-mail: nakano@k.hosei.ac.jp).

Digital Object Identifier 10.1109/TAP.2004.840755 spiral is often backed by a PEC reflector, which changes the inherent bi-directional radiation beam to a unidirectional beam. Conventionally, the antenna height (the distance between the spiral and the PEC plane) is chosen to be one-quarter wavelength. It has already been revealed that, as the frequency decreases from the design frequency, the axial ratio deteriorates [4].

Recently, new antenna structures have been investigated, in which the conventional PEC reflector is replaced with other types of reflectors: corrugated reflectors [5], [6] and electromagnetic band-gap (EBG) reflectors [7]-[11]. For example, a circular corrugated reflector and a perforated EBG reflector (a dielectric substrate drilled with a triangular lattice of circular holes) have been applied to a round helical antenna [5], [6] and an equiangular spiral antenna [11], respectively. The experimental results show that these reflectors are useful in improving the radiation characteristics. However, little data based on theoretical analysis of the helical and spiral antennas with these reflectors are currently available.

In light of this fact, we deal with corrugated and EBG reflectors using theoretical techniques based on the finite-difference time-domain method (FDTDM) [12]. The FDTDM in this paper is formulated using rectangular parallelepiped cells. For easy implementation of the FDTDM, we let the helical and corrugated structures be square, as shown in Fig. 2. Similarly, we let the spiral be square, and adopt an EBG reflector composed of square mushroom-like patches, as shown in Fig. 12.

In Section II, first, the configuration parameters required for a helical antenna backed by a flat PEC reflector to act as an axial-mode CP radiation element are considered. Optimization for a $\mathrm{CP}$ wave radiation element is performed using an infinitesimally thin PEC reflector of infinite extent and, later, a thick PEC reflector of finite extent (finite-sized PEC reflector). Note that the helix with the finite-sized PEC reflector is used as a reference helical antenna in this paper.

Second, the finite-sized PEC reflector of the reference helical antenna is transformed to a corrugated reflector. The radiation characteristics of the helix backed by the corrugated reflector are analyzed and discussed. The current flowing over the rear surface of the corrugated reflector is compared with that of the finite-sized PEC reflector. It is found that the corrugated reflector contributes to reduction in the backward radiation. 
In Section III, first, a spiral antenna backed by a PEC reflector is analyzed. This spiral antenna is referred to as a PEC spiral antenna. The PEC spiral has an outermost peripheral length of more than one wavelength but less than two wavelengths. This peripheral length is the length required to support first mode $\mathrm{CP}$ radiation [3]. The PEC spiral is optimized to radiate a $\mathrm{CP}$ wave at an antenna height of one-quarter wavelength. The analysis reveals that, as the antenna height decreases from one-quarter wavelength, the cross-polarization component increases.

Second, the PEC reflector for the spiral is replaced with an EBG reflector composed of mushroom-like elements [8]-[10]. The spiral backed by the mushroom-like EBG reflector (M-EBG reflector) is referred to as an M-EBG spiral antenna in this paper. The analysis of the M-EBG spiral is performed for a small antenna height: the distance between the spiral and the M-EBG surface is 0.06 wavelength. It is revealed that the use of the $\mathrm{M}-\mathrm{EBG}$ reflector reduces the cross-polarization component. In other words, the spiral radiates a CP wave with a small antenna height with the help of the M-EBG reflector.

Throughout this paper, the analysis is performed using FDTDM computer programs developed by the authors. Some of the FDTDM results are compared with those obtained by experiments.

\section{HelicAl ANTENNAS}

In this section, two types of conducting reflectors are used to back a helical arm: one is a flat PEC reflector and the other is a corrugated reflector. A comparison between the radiation patterns for these two reflectors reveals that the corrugated reflector is a useful tool in reducing the backward radiation. Note that the comparison is performed under the condition that both the reflectors are finite and have the same size.

\section{A. Helical Antenna Above a Flat PEC Reflector (Determination of a Reference Helical Antenna)}

For analysis, we use the FDTDM based on Yee's cells, with each rectangular parallelepiped having side lengths $\Delta X, \Delta Y$, and $\Delta Z$. If the FDTDM is applied to a curved helical arm, numerical error results from the staircase approximation of the curved arm. To avoid this error, we adopt a square helical arm. (For this same reason, all antennas and reflectors treated in this paper are based on square or rectangular structures.)

The cell side lengths used in this paper are between $\lambda / 66$ and $\lambda / 200$ ( $\lambda$ being wavelength). These cell side lengths and the time step $\Delta t$ satisfy Courant's condition [12] for stability of the FDTDM. The analysis space is truncated with Liao's secondorder absorbing boundary condition [13]. Based on the electric and magnetic fields obtained using the FDTDM, we calculate the antenna characteristics, including the radiation pattern, axial ratio, input impedance, and gain [14].

Fig. 1 shows the configuration of a helical antenna above a flat PEC reflector. The helix is specified by the virtual circumference $4 s$, first vertical length $h_{f}$, pitch $p$ (the distance from points a to b), and arm length $s_{\text {arm }}$. The helix is excited from feed point $P_{F}$. The flat PEC reflector (ground plane) is square and is specified by the side length $g$ and thickness $T_{g}$.

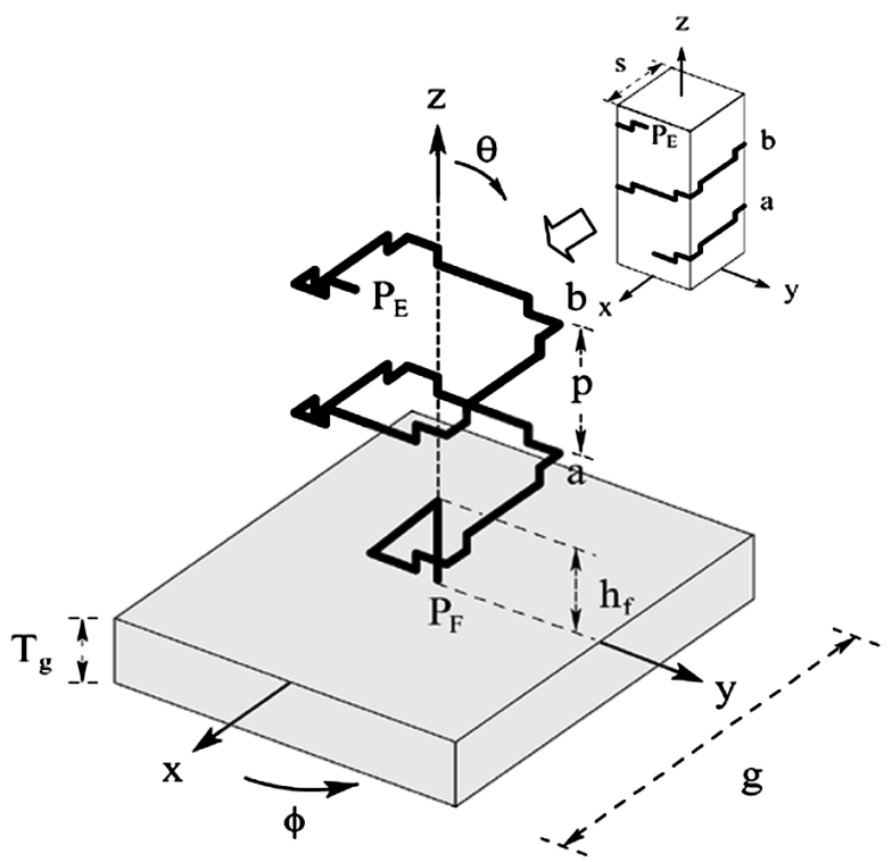

(a)

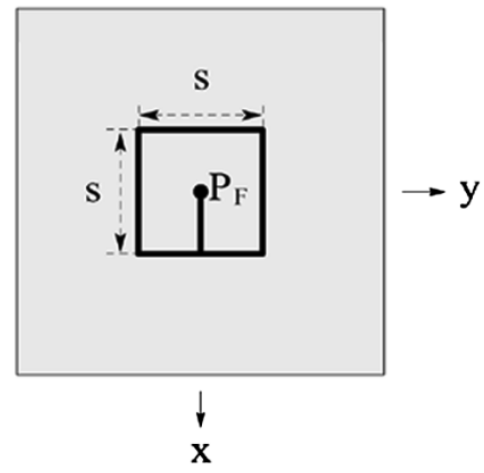

(b)

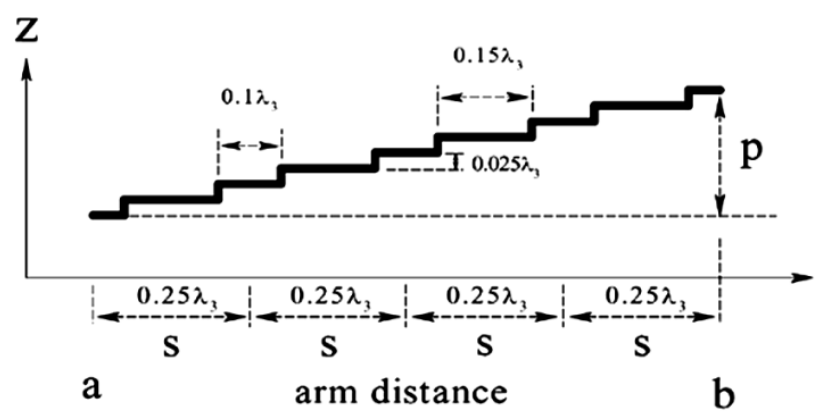

(c)

Fig. 1. Configuration of an axial-mode helical antenna backed by a flat PEC reflector. (a) Perspective view. (b) Top view. (c) Helical arm from points a to b.

To obtain a $\mathrm{CP}$ axial-beam, the virtual circumference $4 \mathrm{~s}$ is chosen to be one wavelength at a test frequency of $3 \mathrm{GHz}(4 s=$ $1.0 \lambda_{3}$, with $\lambda_{3}$ being the free-space wavelength at $3 \mathrm{GHz}$ ). The first vertical length and pitch are arbitrarily chosen to be $h_{f}=$ $0.1 \lambda_{3}$ and $p=0.2 \lambda_{3}$, respectively. The helical arm length $s_{\text {arm }}$, side length $g$, and thickness $T_{g}$ are varied subject to the objectives of the analysis, holding the major helix parameters at $\left(4 s, p, h_{f}\right)=\left(1.0 \lambda_{3}, 0.2 \lambda_{3}, 0.1 \lambda_{3}\right) \equiv\left(\mathrm{HLX}_{0}\right)$. 


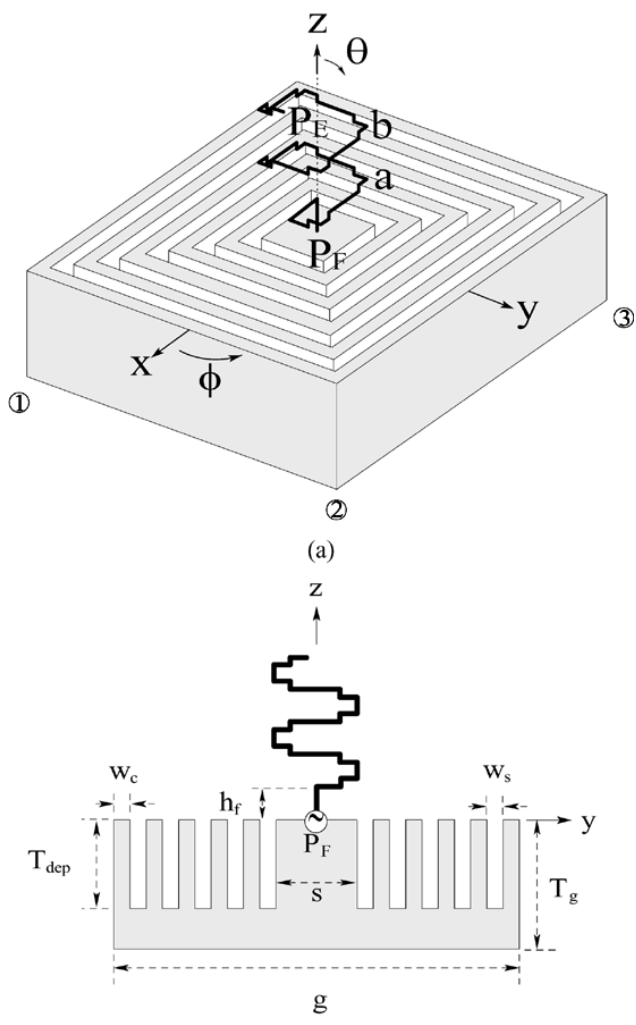

(b)

Fig. 2. Configuration of an axial-mode helical antenna backed by a corrugated reflector. (a) Perspective view. (b) Side view.

The analysis in [15] reveals that a long axial-mode helix has two distinct arm regions: an arm region between the feed point and the current minimum point (approximately the first two turns near the feed point), and the remaining arm region. The former and latter, respectively, act as a CP wave exciter and a $\mathrm{CP}$ wave director. This means that $\mathrm{CP}$ wave radiation is obtained with a relatively small arm length (corresponding to a small number of turns [16]).

We start with optimization of the helical arm length $s_{\text {arm }}$ to obtain $\mathrm{CP}$ wave radiation. The optimization is performed under the conditions that the flat PEC reflector has an infinite side length $(g=\infty)$ and is infinitesimally thin $\left(T_{g}=0\right)$. It is found that a CP wave is obtained at $s_{\text {arm }} \approx 2.6 \lambda_{3} \equiv s_{\text {opt }}$, which forms an approximately two-turn arm. Based on this result, the helical arm parameters are fixed to be $\left(\left(4 s, p, h_{f}\right), s_{\text {arm }}\right)=$ $\left(\left(\mathrm{HLX}_{0}\right), s_{\text {opt }}\right)=\left(\left(1.0 \lambda_{3}, 0.2 \lambda_{3}, 0.1 \lambda_{3}\right), 2.6 \lambda_{3}\right) \equiv$ HLX.

Next, the side length of the flat PEC reflector, $g$, is varied for a helix with the configuration parameters HLX (the HLX helix), holding the reflector thickness at $T_{g}=0$ (infinitesimally thin). The current induced on this finite-sized flat PEC reflector affects the antenna characteristics. The analysis shows that the gain varies with the reflector size, showing the maximum gain at $g / s \approx 5$. Based on this result, we fix the side length to be $g=5 s=1.25 \lambda_{3} \equiv g_{\max }$.

Finally, the thickness of the flat PEC reflector, $T_{g}$, is varied for the HLX helix, holding the side length of the reflector at $g=g_{\max }$, that is, $\left(T_{g}, g\right)=$ (variable, $\left.1.25 \lambda_{3}\right)$. The analysis shows that the effect of the thickness $T_{q}$ on the gain and axial ratio is not significant. To corrugate the flat $\mathrm{PEC}$ reflector, which

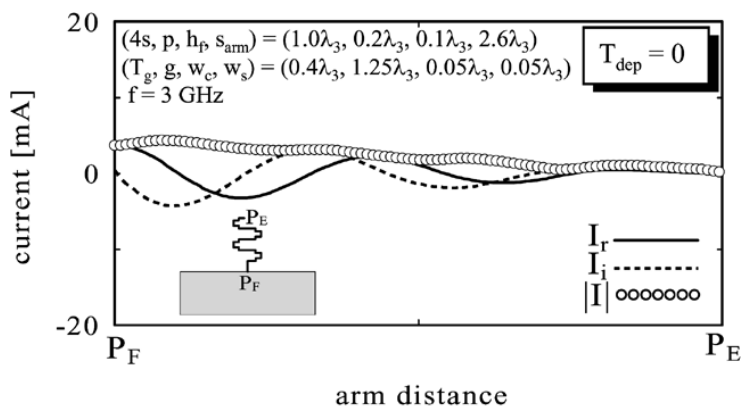

(a)

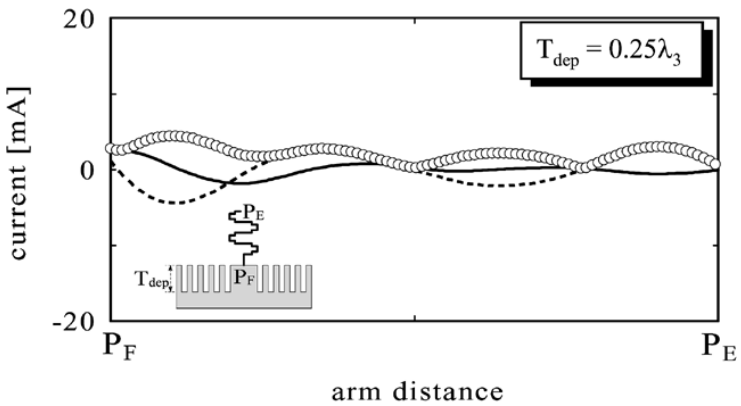

(b)

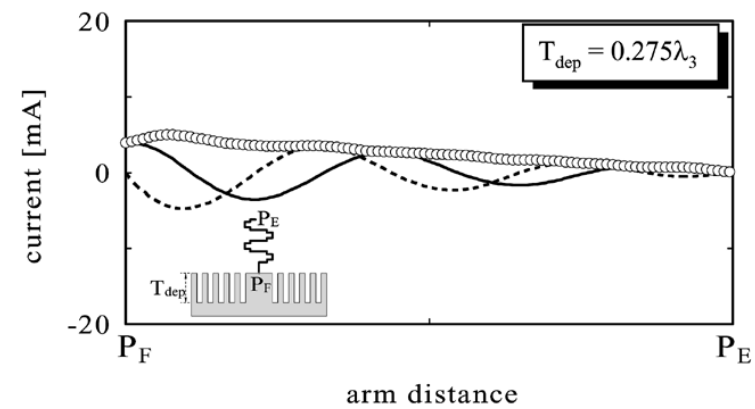

(c)

Fig. 3. Current distribution as a function of corrugation depth $T_{\text {dep }}$.

is used in Section II-B, we choose the reflector thickness to be more than one-quarter wavelength: $T_{g}=0.4 \lambda_{3} \equiv T_{\mathrm{G}}$. Note that the HLX helix backed by a flat PEC reflector of $\left(T_{g}, g\right)=$ $\left(T_{\mathrm{G}}, g\right)=\left(0.4 \lambda_{3}, 1.25 \lambda_{3}\right)$ is designated as the reference helical antenna for the following discussion.

\section{B. Helical Antenna Above a Corrugated Reflector}

A corrugated structure composed of $x$-directed ridges and slots (cut into a metal slab, whose top surface coincides with the $x-y$ plane of the rectangular coordinate system) has the following characteristics [8]: 1) if there are many slots per wavelength, the structure can be assigned an effective surface impedance equal to the impedance of the slots; 2) if the slot depth is greater than one-quarter wavelength, the surface impedance is capacitive, and TM surface waves cannot exist; that is, TM surface waves do not propagate in the $y$ direction.

We transform the aforementioned one-directional corrugated structure ( $x$-directed ridges and slots) to a coaxial corrugated structure. Fig. 2 shows such a structure, where the PEC reflector of the reference helical antenna is coaxially corrugated. The corrugation is specified by the conductor width (ridge width) $w_{c}$, slot width (groove width) $w_{s}$, and depth $T_{\text {dep }}$. Note that 


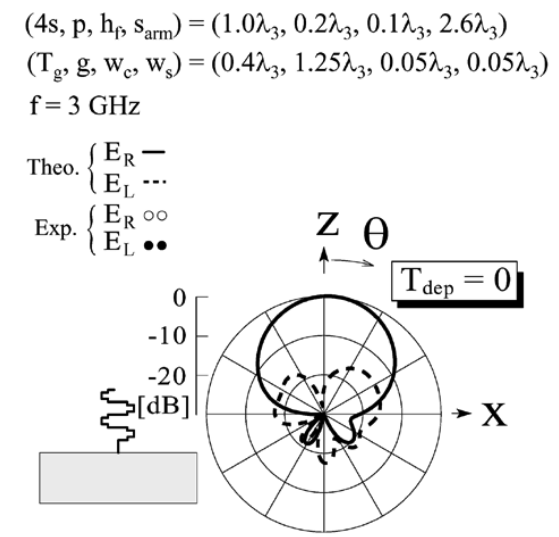

(a)

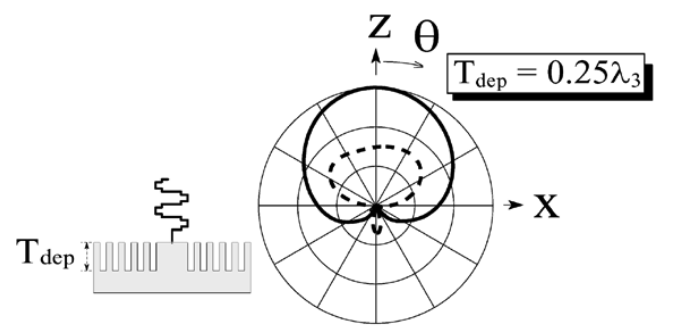

(b)

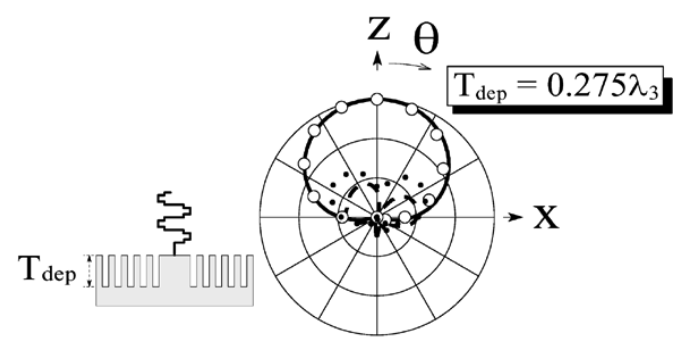

(c)

Fig. 4. Radiation pattern as a function of corrugation depth $T_{\text {dep }}$.

the shape of helix remains unchanged, having the configuration parameters HLX determined in Section II-A above, that is, $\left(4 s, p, h_{f}, s_{\text {arm }}\right)=\left(1.0 \lambda_{3}, 0.2 \lambda_{3}, 0.1 \lambda_{3}, 2.6 \lambda_{3}\right)$.

The corrugation depth $T_{\text {dep }}$ affects the current distribution along the helical arm. Fig. 3 shows the current distribution as a function of $T_{\text {dep }}$, where the conductor width $w_{c}$ and slot width $w_{s}$ are fixed to be $\left(w_{c}, w_{s}\right)=(s / 5, s / 5)=\left(0.05 \lambda_{3}, 0.05 \lambda_{3}\right)$. It is found that there is a depth at which a traveling wave current with a smooth decay along the helical arm is obtained, as shown in Fig. 3(c), where $T_{\text {dep }}=0.275 \lambda_{3} \equiv T_{\text {DEP }}$.

The radiation pattern for a corrugation depth of $T_{\mathrm{dep}}=T_{\mathrm{DEP}}$ (for which the current distribution shown in Fig. 3(c) is obtained) is presented in Fig. 4(c), together with the patterns for $T_{\text {dep }}=0$ and $T_{\text {dep }}=0.25 \lambda_{3}$ (for which the current distributions shown in Fig. 3(a) and (b) are obtained, respectively). Each radiation field is decomposed into a right-hand $\mathrm{CP}$ wave component $\left(E_{R}\right)$ and a left-hand CP wave component $\left(E_{L}\right)$. It is found that the helix with $T_{\text {dep }}=T_{\mathrm{DEP}}=0.275 \lambda_{3}$ has the smallest cross-polarization component and backward radiation (in the negative $z$ direction) of the three helices. For confirmation, the experimental results are presented in Fig. 4(c). The experimental results are in good agreement with the theoretical results.

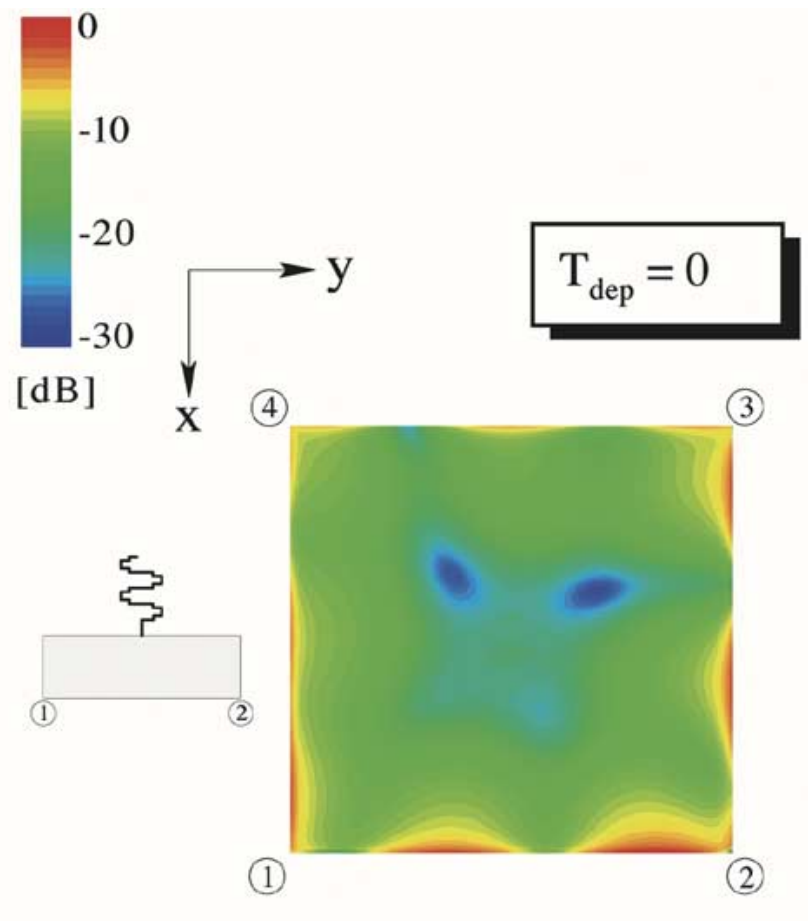

(a)

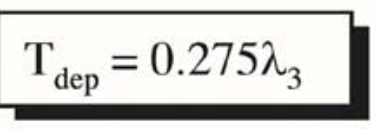

(4)

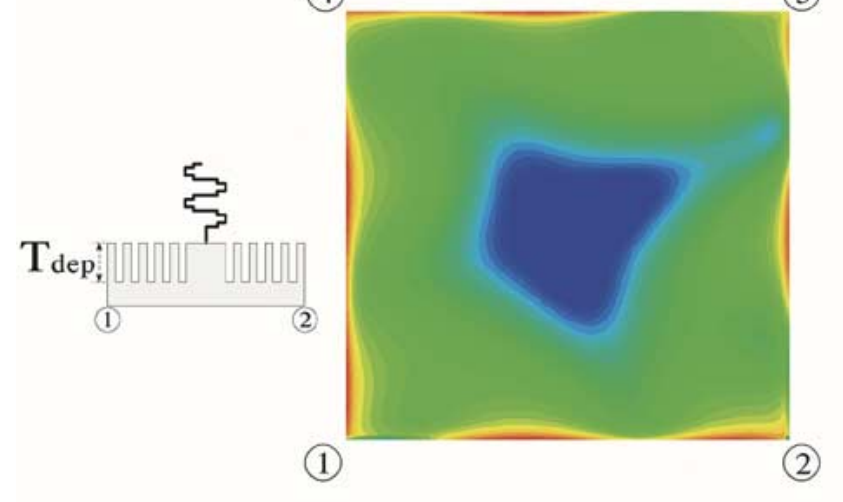

(b)

Fig. 5. Rear surface currents. (a) Flat PEC reflector. (b) Corrugated reflector.

The backward radiation is closely related to the currents induced over the reflector surface. A detailed investigation reveals that the currents on the corrugated reflector with $T_{\text {dep }}=$ $T_{\mathrm{DEP}}=0.275 \lambda_{3}$ are concentrated on the ridges near the helix. It follows that the currents flowing toward the rear surface (at $z=-T_{\mathrm{G}}=-0.4 \lambda_{3}$ ) of the corrugated reflector are small. Fig. 5 shows a comparison between the theoretical current densities (each expressed by $|\mathbf{J}|$, where $\mathbf{J}=J_{x} \hat{x}+J_{y} \hat{y}$ ) over the rear surfaces of the flat PEC and the corrugated reflectors. It is clear that the current density over the rear surface of the corrugated reflector is smaller than that of the flat PEC reflector. In 


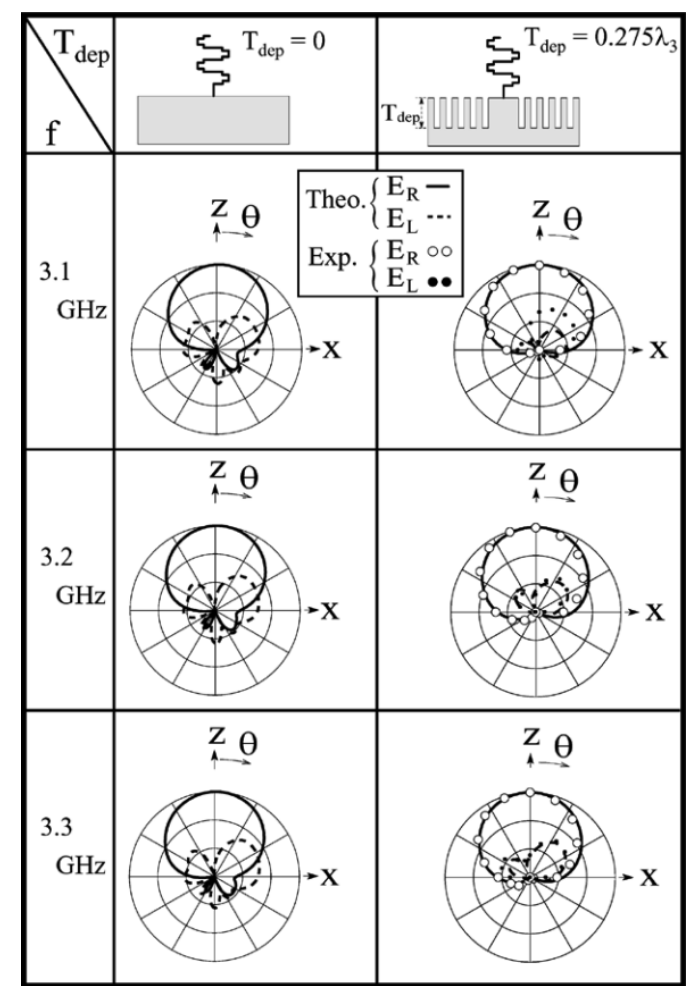

Fig. 6. Radiation pattern as a function of frequency.

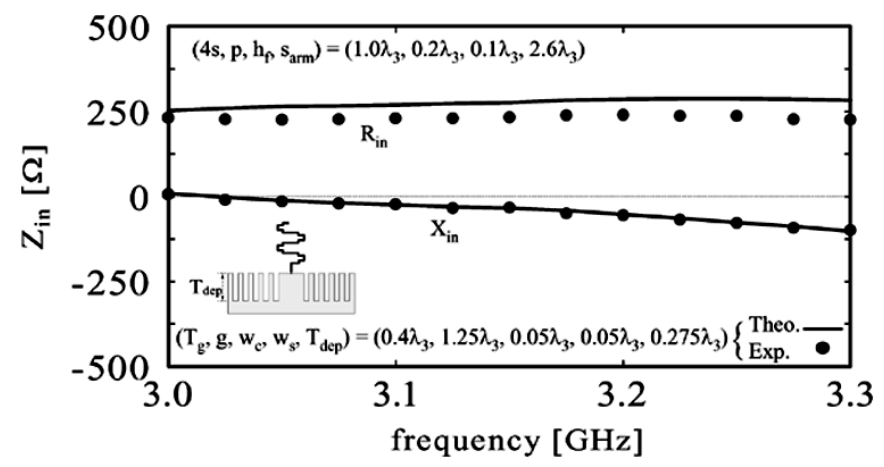

Fig. 7. Input impedance as a function of frequency.

other words, the corrugation suppresses the current flowing toward the rear surface of the reflector, resulting in a reduction in the backward radiation.

So far, we have discussed the antenna characteristics using a test frequency of $3 \mathrm{GHz}$. Next, we consider the frequency response, focusing on the HLX helix backed by a corrugated reflector of $\left(T_{g}, g, w_{c}, w_{s}, T_{\mathrm{dep}}\right)=\left(T_{\mathrm{G}}, g_{\max }, s / 5, s / 5, T_{\mathrm{DEP}}\right)=$ $\left(0.4 \lambda_{3}, 1.25 \lambda_{3}, 0.05 \lambda_{3}, 0.05 \lambda_{3}, 0.275 \lambda_{3}\right)$. The analysis over a frequency band of 3 to $3.3 \mathrm{GHz}$ ( $9.5 \%$ bandwidth) reveals that the backward radiation for the corrugated reflector is always smaller than that for the flat PEC reflector (see Fig. 4(a) and (c) at $3 \mathrm{GHz}$ and Fig. 6). Within this frequency band, the gain $\mathrm{G}_{R}$ for a right-hand CP wave is approximately $9 \mathrm{dBi}$ and the input impedance $\left(Z_{\text {in }}=R_{\text {in }}+j X_{\text {in }}\right)$ is relatively constant, as shown in Fig. 7. Note that the theoretical radiation patterns and input impedance for the HLX helix backed by a corrugated

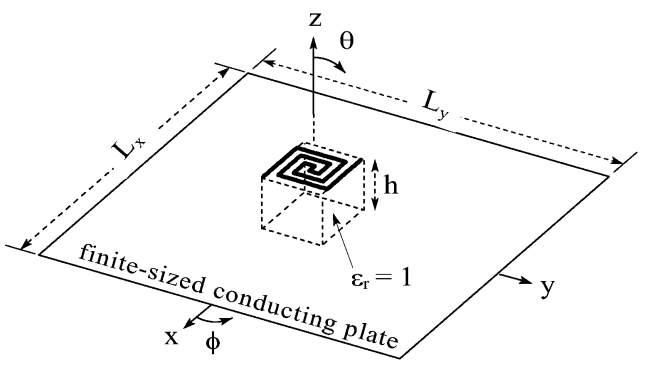

(a)

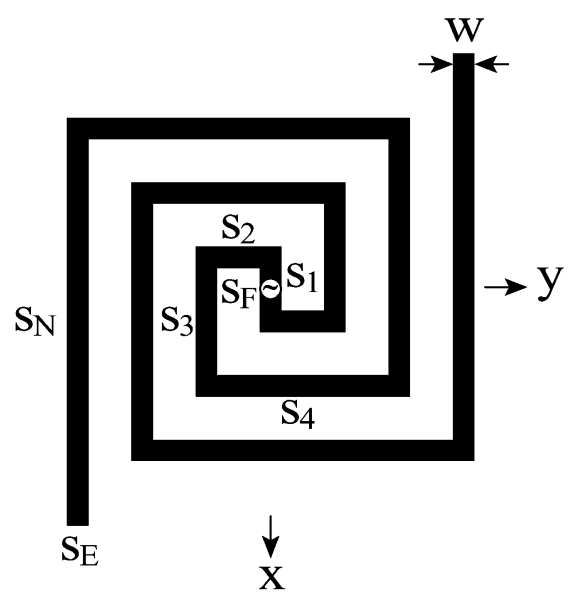

(b)

Fig. 8. Spiral antenna backed by a flat PEC reflector. (a) Perspective view. (b) Spiral arms.

reflector, shown in Figs. 6 and 7, respectively, are confirmed by the experimental results.

\section{SPIRAL ANTENNAS}

A PEC reflector is often used with a spiral antenna to radiate a uni-directional beam. Effects of reducing the antenna height (the distance between the spiral and the PEC plane) on the radiation characteristics are discussed in Section III-A below. The degraded radiation beam for a small antenna height, described in Section III-A, is restored to a desirable unidirectional CP radiation beam in Section III-B, where a mushroom-like EBG reflector (M-EBG reflector) is used.

\section{A. Spiral Antenna Above a Flat PEC Reflector (PEC Spiral Antenna)}

Fig. 8 shows a spiral antenna backed by an infinitesimally thin flat PEC reflector, where the distance between the spiral and the PEC plane is denoted by $h$. The spiral in Fig. 8 is referred to as a PEC spiral antenna in this paper.

The spiral has two arms of width $w$, each being composed of $N$ elements. The element lengths are: $s_{1}$ (first element length) and $s_{i}=2(i-1) s_{1}$ for $i=2,3, \ldots N$. The peripheral length $C_{\text {sprl }}$, defined by $4 s_{N}$, is chosen to be $1.44 \lambda_{6}$ with $s_{1}=0.03 \lambda_{6}$ and $N=7$, where $\lambda_{6}$ is the wavelength at a test frequency of 6 GHz. This spiral supports first mode radiation at $6 \mathrm{GHz}$ [3].

Fig. 9 shows the radiation pattern of the PEC spiral, where the antenna heights in Fig. 9(a) and (b) are chosen to be $h=$ $(1 / 4) \lambda_{6}$ and $(1 / 10) \lambda_{6}$, respectively, and the sizes of the flat 

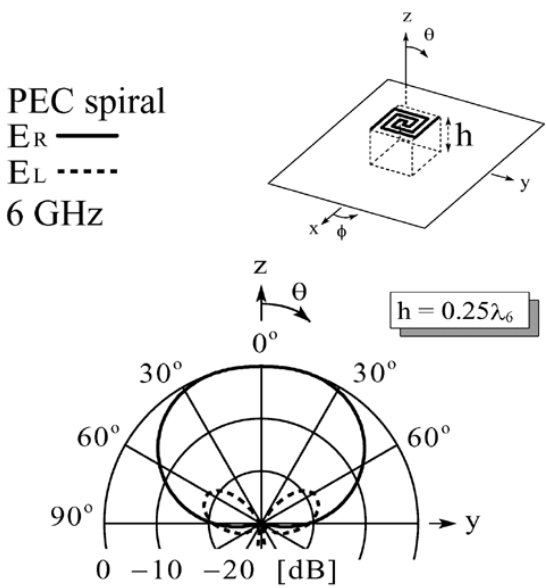

(a)

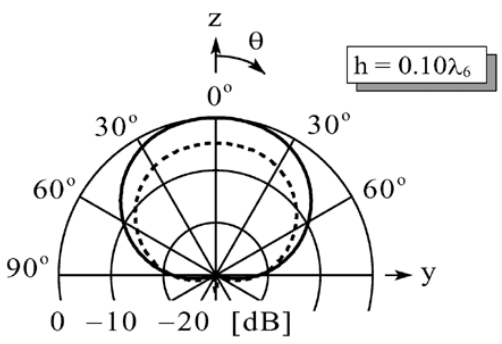

(b)

Fig. 9. Radiation patterns of a PEC spiral antenna at different antenna heights. (a) $h=(1 / 4)$ wavelength at $6 \mathrm{GHz}$. (b) $h=(1 / 10)$ wavelength at $6 \mathrm{GHz}$.

PEC reflectors for Fig. 9(a) and (b) are the same $\left(L_{x}=L_{y}=\right.$ $218 \Delta=2.18 \lambda_{6}$, where $\Delta=0.01 \lambda_{6}$ ).

As seen from Fig. 9(a), the spiral radiates a CP wave at $h=$ $(1 / 4) \lambda_{6}$, as desired. The cross-polarization component is small and the axial ratio (AR) in the $z$ direction is calculated to be 0.6 $\mathrm{dB}$. In other words, the spiral is optimized to radiate a CP wave. It is, however, noted that this desired $\mathrm{CP}$ radiation deteriorates when the antenna height $h$ is made smaller than one-quarter wavelength, as shown in Fig. 9(b), where the axial ratio in the $z$-axis direction is undesirably high: $11.3 \mathrm{~dB}$. The high axial ratio is due to an increased reverse current flowing toward the feed point. Note that the reverse current generates a cross-polarization component (left-hand $\mathrm{CP}$ wave component $E_{L}$ ).

The deteriorated axial ratio must be improved for the spiral to operate as a CP antenna. For this, the flat PEC reflector is replaced with an M-EBG reflector in the following section.

\section{B. Spiral Antenna Above an M-EBG Reflector (M-EBG Spiral Antenna)}

As shown in Fig. 10, the M-EBG reflector used in this section consists of mushroom-like elements [8]-[10], composed of numerous patches, each being connected to a bottom conducting plate (ground plate) using a conducting pin [8]-[10]. The patch, printed on a dielectric substrate of relative permittivity $\varepsilon_{r}$, is square with side length $s_{\text {patch }}$. The conducting pin is located at the center of the patch. The thickness of the M-EBG reflector (i.e., the pin length) is denoted by $B$. The numbers of patches in the $x$ and $y$ directions are $N_{x}$ and $N_{y}$, respectively, and the space between the neighboring patches is denoted by $\delta$.

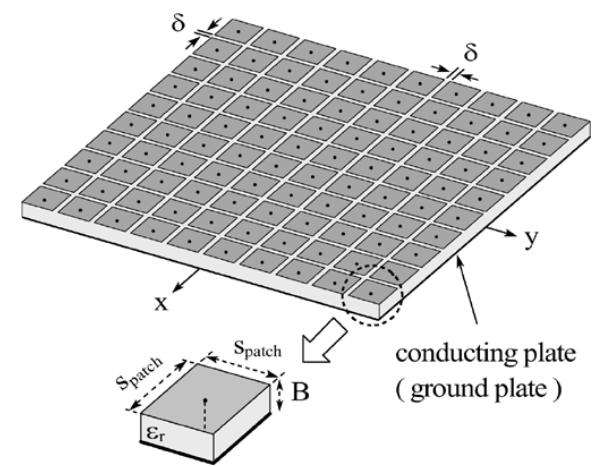

Fig. 10. EBG structure with mushroom-like elements.

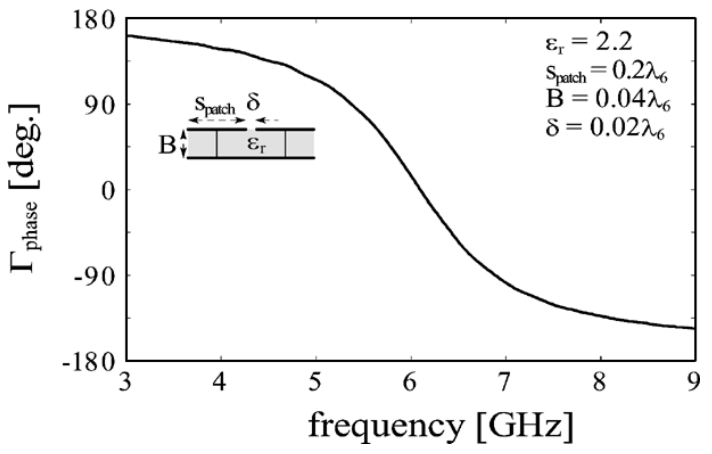

Fig. 11. Reflection phase $\Gamma_{\text {phase }}$ as a function of frequency.

Interaction between an electromagnetic incident plane wave and the M-EBG reflector yields a capacitance $C$ and an inductance $L$ : the proximity of the neighboring patches creates capacitance, while the currents flowing between the top patches and the bottom plate through the conducting pins creates inductance. The surface impedance of the M-EBG reflector is modeled by a parallel resonant circuit (with impedance $Z=$ $\left.j \omega L /\left(1-\omega^{2} L C\right)\right)$ [8]. The impedance is high around the resonant frequency and an incident plane wave is reflected in-phase, rather than out-of-phase. This means that the M-EBG reflector behaves similarly to a perfect magnetic conductor (PMC).

We design the M-EBG reflector such that it resonates at 6 $\mathrm{GHz}$ (the test frequency used in the previous Section III-A) using $\varepsilon_{r}=2.2, s_{\text {patch }}=0.2 \lambda_{6}, \mathrm{~B}=0.04 \lambda_{6}$ and $\delta=0.02 \lambda_{6}$. Fig. 11 shows the reflection phase $\Gamma_{\text {phase }}$ when an electromagnetic plane wave illuminates the M-EBG reflector, where $\Gamma_{\text {phase }}$ is defined as the phase of the reflected electric field at the reflecting surface. As seen from this figure, the M-EBG reflector has a reflection phase of $\Gamma_{\text {phase }} \approx 0^{\circ}$ at $6 \mathrm{GHz}$, realizing the characteristic of a PMC. Note that $\Gamma_{\text {phase }}$ is obtained under the condition that the number of patches is $N_{x}=N_{y}=\infty$. Also, note that the authors' computer programs are able to reproduce Fig. 5 in [9], thereby confirming the correctness of the authors' computer programs.

Next, we counteract the deterioration in the radiation shown in Fig. 9(b) to restore the $\mathrm{CP}$ wave radiation, while leaving the spiral configuration parameters unchanged. This is performed by replacing the flat PEC reflector in Fig. 8 with the M-EBG reflector, as shown in Fig. 12. The spiral backed by the M-EBG reflector is hereafter referred to as the M-EBG spiral antenna, 


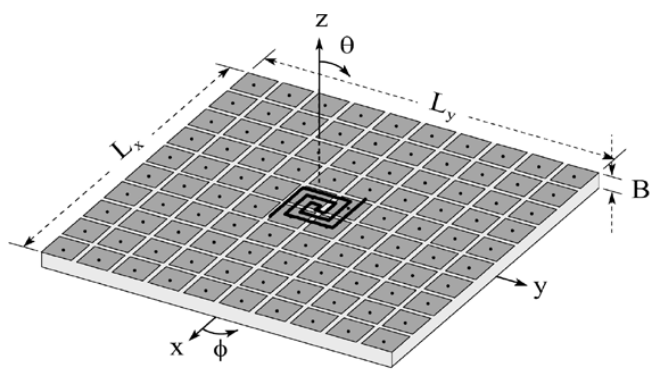

(a)

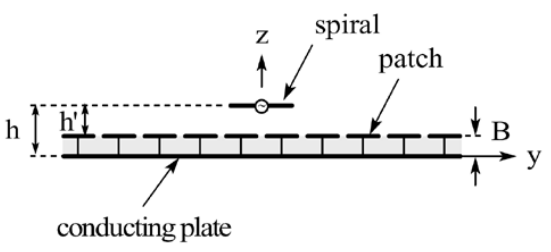

(b)

Fig. 12. Spiral antenna backed by an M-EBG reflector. (a) Perspective view. (b) Side view.

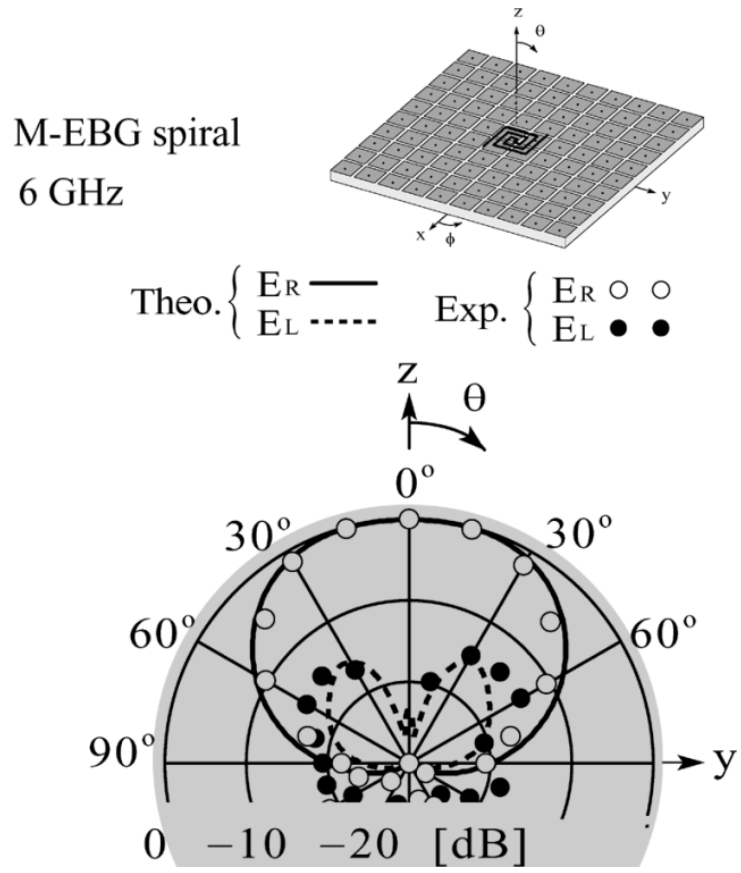

Fig. 13. Radiation pattern for an M-EBG spiral antenna, where the antenna height is $h=(1 / 10)$ wavelength at $6 \mathrm{GHz}$.

which contrasts with the PEC spiral antenna in Section III-A above.

The current along the M-EBG spiral arms and the image current (parallel to the M-EBG reflector surface) at $6 \mathrm{GHz}$ are in-phase. This allows us to lower the antenna height. We locate the radiation elements (antenna arms) of the M-EBG spiral at the same height $\left(h=(1 / 10) \lambda_{6}\right.$ from the ground plate) as those of the PEC spiral described in Section III-A. It should be noted that the distance from the M-EBG reflector surface to the radiation elements, $h^{\prime}(=h-\mathrm{B})$, is very small: $h^{\prime}=0.06 \lambda_{6}$. Also, note that the M-EBG reflector has the same side length as the conducting plate used for the PEC spiral: $L_{x}=L_{y}=2.18 \lambda_{6}$

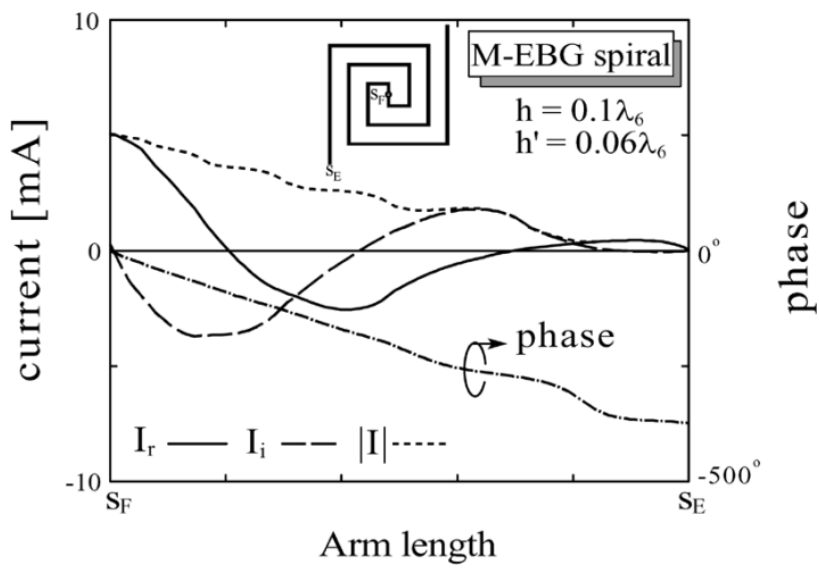

(a)

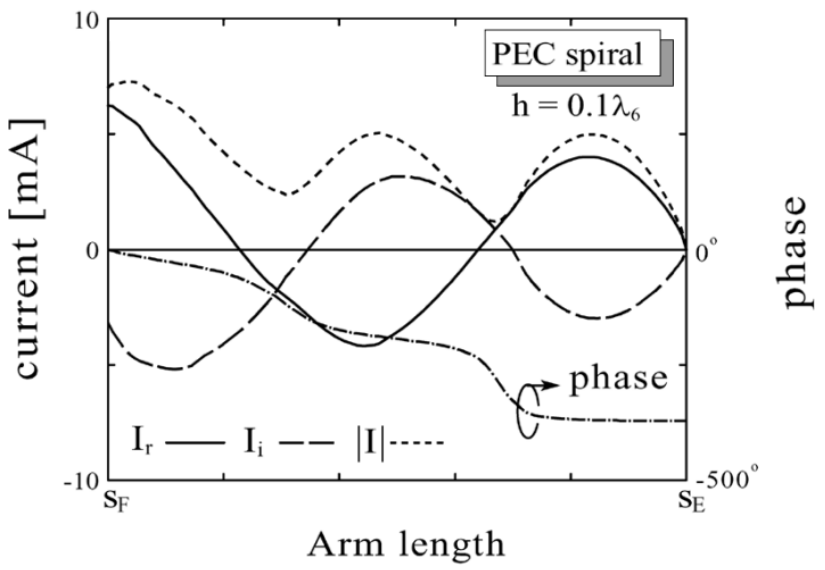

(b)

Fig. 14. Current distributions at $6 \mathrm{GHz}$. (a) Spiral backed by an M-EBG reflector. (b) Spiral backed by a PEC reflector.

with $N_{x}=N_{y}=10$. Thus, the M-EBG spiral and the PEC spiral can be fairly compared.

Fig. 13 shows the theoretical radiation pattern of the M-EBG spiral antenna at $6 \mathrm{GHz}$, together with experimental results. We can see clearly from this figure that $\mathrm{CP}$ radiation has been restored for the M-EBG spiral, while maintaining a small antenna height of $h=(1 / 10) \lambda_{6}$. The theoretical axial ratio is approximately $1.2 \mathrm{~dB}$ in the $z$ direction. The restoration of CP radiation is attributed to the fact that the current on each spiral arm is a traveling wave with smooth decay, as shown in Fig. 14(a), in contrast with the current of the PEC spiral, shown in Fig. 14(b), which does not decay.

So far, the radiation characteristics at $6 \mathrm{GHz}$ have been discussed. Now we investigate the frequency response of the M-EBG spiral in the vicinity of $6 \mathrm{GHz}$. Fig. 15 shows the theoretical axial ratio in the $z$ direction as a function of frequency (the experimental axial ratio is also presented in this figure). The bandwidth for a 3-dB axial ratio criterion is calculated to be $11 \%$. Detailed investigation shows that, as the frequency is increased in the 3-dB axial ratio bandwidth, the half-power beam width (HPBW) increases. It follows that the gain decreases, as shown in Fig. 16. (Note that the theoretical and experimental gains in Fig. 16 are in good agreement.) The theoretical gain variation is $4.1 \mathrm{~dB}$ within an axial ratio bandwidth of $11 \%$. 


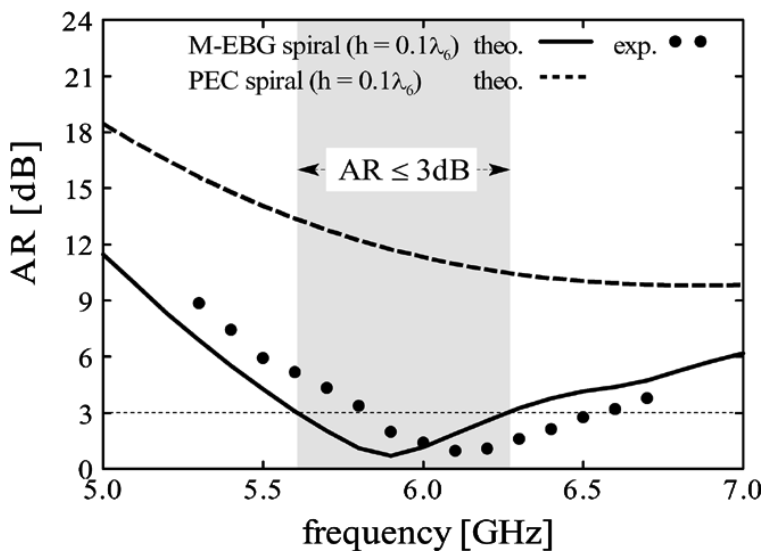

Fig. 15. Axial ratio (AR) as a function of frequency.

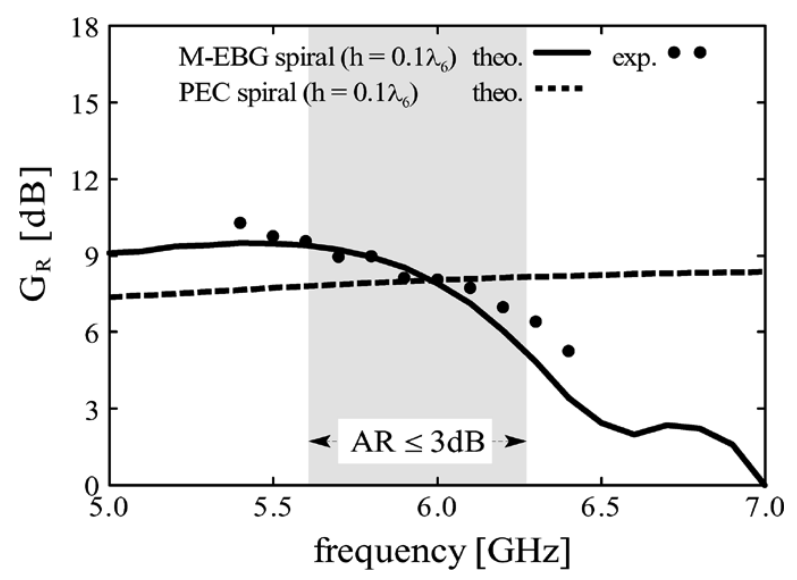

Fig. 16. Gain for a right-hand $\mathrm{CP}$ wave, $\mathrm{G}_{R}$, as a function of frequency.

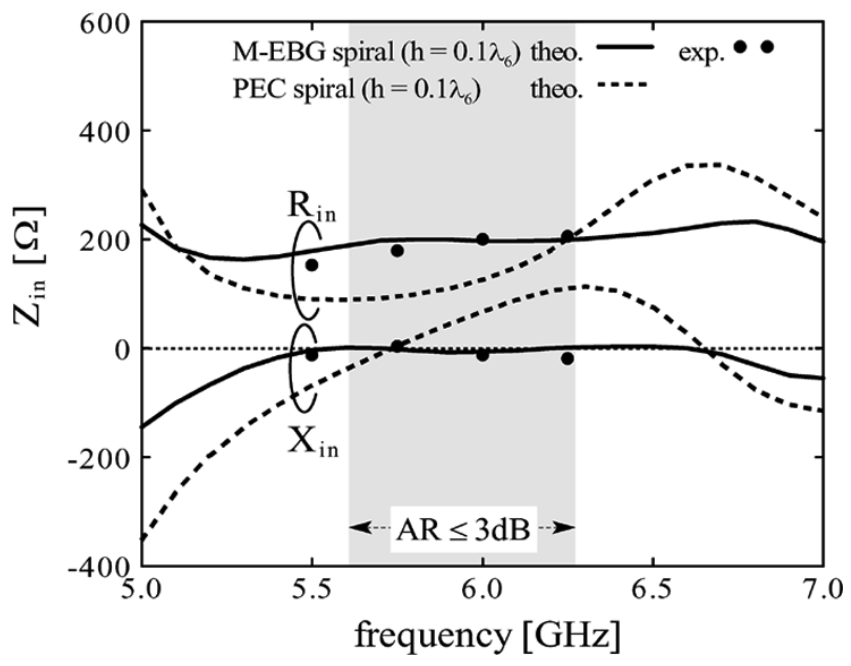

Fig. 17. Input impedance $Z_{\text {in }}=R_{\text {in }}+j X_{\text {in }}$ as a function of frequency.

Fig. 17 shows the input impedance of the M-EBG spiral as a function of frequency. For comparison, the impedance of the PEC spiral is also illustrated in this figure. It is worth mentioning that the M-EBG spiral has a more stable input impedance over a wide frequency bandwidth (including the 3-dB axial ratio bandwidth) than the PEC spiral. Also note that the FDTDM input impedance results for the M-EBG spiral are confirmed by the experimental results.

\section{CONCLUSION}

Helical and spiral antennas backed by corrugated and M-EBG reflectors, respectively, have been investigated after optimizing both the antennas' configurations in the presence of flat PEC reflectors.

The helical antenna, whose virtual circumference is given as $4 s$, has been analyzed under the condition that a flat square PEC reflector of side length $g$ backs the helix. It is found that the gain varies with the PEC reflector size, showing maximum gain at $g / s \approx 5$. It is also found that the effects of the thickness $T_{g}$ of the finite-sized PEC reflector (of $g / s \approx 5$ ) on the gain and axial ratio are not significant. Based on these results, a flat PEC reflector with $g=5 s$ and $T_{g}=0.4 \lambda_{3}$ is transformed to a corrugated reflector for backing the same helix. A comparison between the currents over the rear surfaces of the corrugated and flat PEC reflectors reveals that the corrugated reflector contributes to reduction in the backward radiation.

A spiral antenna, whose peripheral length is more than one wavelength, but less than two wavelengths, is optimized for first-mode CP radiation in the presence of a flat finite PEC reflector. The optimization is performed for an antenna height of one-quarter wavelength. As the antenna height decreases, the axial ratio for the spiral deteriorates. Improvement in the axial ratio at a lower antenna height is investigated using a spiral backed by an M-EBG reflector. For this investigation, a representative antenna height of $h=0.1 \lambda_{6}$ (the distance from the M-EBG surface to the spiral is extremely small: $h^{\prime}=0.06 \lambda_{6}$ ) is used. It is found that the spiral backed by the M-EBG reflector radiates a $\mathrm{CP}$ wave, as desired. It is also revealed that the input impedance for the M-EBG reflector is more stable over a wide frequency band than that for the PEC reflector.

\section{ACKNOWLEDGMENT}

The authors thank V. Shkawrytko for his kind assistance in the preparation of this manuscript.

\section{REFERENCES}

[1] J. D. Kraus, Antennas, 2nd ed. New York: McGraw-Hill, 1988, ch. 7

[2] H. Nakano, J. Yamauchi, and H. Mimaki, "Backfire radiation from a monofilar helix with a small ground plane," IEEE Trans. Antennas Propag., vol. 36, no. 10, pp. 1359-1364, Oct. 1988.

[3] J. A. Kaiser, "The Archimedean two-wire spiral antenna," IRE Trans. Antennas Propagat., vol. AP-8, pp. 312-323, May 1960.

[4] H. Nakano, K. Nogami, S. Arai, H. Mimaki, and J. Yamauchi, "A spiral antenna backed by a conducting plane reflector," IEEE Trans. Antennas Propag., vol. AP-34, no. 6, pp. 791-796, Jun. 1986.

[5] Z. Ying and P. S. Kildal, "Improvements of dipole, helix, spiral, microstrip patch, and aperture antennas with ground planes by using corrugated soft surfaces," in Proc. Inst. Elect. Eng. -Microw. Antennas Propag., vol. 143, Jun. 1996, pp. 244-248.

[6] - "Reduced sidelobes and cross-polarization of axial mode helix using a soft corrugated ground plane," in Proc. IEEE AP-S Symp., Ann Arbor, MI, 1993, pp. 462-465.

[7] F. R. Yang, K. P. Ma, Y. Qian, and T. Itoh, "A uniplanar compact photonic-bandgap (UC-PBG) structure and its applications for microwave circuit," IEEE Trans. Microwave Theory Tech., vol. 47, no. 8, pp. 1509-1514, Aug. 1999.

[8] D. Sievenpiper, L. Zhang, R. F. J. Broas, N. G. Alexopoulos, and E. Yablonovitch, "High-impedance electromagnetic surfaces with a forbidden frequency band," IEEE Trans. Microw. Theory Tech., vol. 47, no. 11, pp. 2059-2074, Nov. 1999. 
[9] F. Yang and Y. Rahmat-Samii, "Reflection phase characterizations of the EBG ground plane for low profile wire antenna applications," IEEE Trans. Antennas Propag., vol. 51, no. 10, pp. 2691-2703, Oct. 2003.

[10] - "A low profile circularly polarized curl antenna over electromagnetic band-gap (EBG) surface," Microwave Opt. Technol. Lett., vol. 31, no. 4, pp. 264-267, Nov. 2001.

[11] T. H. Liu, W. X. Zhang, M. Zhang, and K. F. Tsang, "Low profile spiral antenna with PBG substrate," Electron. Lett., vol. 36, no. 9, pp. 779-780, Apr. 2000.

[12] A. Taflove, Computational Electrodynamics: The Finite-Difference Time Domain Method. Norwood, MA: Artech House, 1995.

[13] Z. P. Liao, H. L. Wong, B. P. Yang, and Y. F. Yuan, "A transmitting boundary for transient wave analysis," Science Sinica, ser. A, vol. 27, no. 10, pp. 1063-1076, 1984.

[14] H. Nakano, M. Ikeda, K. Hitosugi, and J. Yamauchi, "A spiral antenna sandwiched by dielectric layers," IEEE Trans. Antennas Propag., vol. 52, no. 6, pp. 1417-1423, Jun. 2004.

[15] H. Nakano and J. Yamauchi, "Radiation characteristics of helix antenna with parasitic elements," Electron. Lett., vol. 16, no. 18, pp. 687-688, Aug. 1980.

[16] H. Nakano, H. Takeda, T. Honma, H. Mimaki, and J. Yamauchi, "Extremely low-profile helix radiating a circularly polarized wave," IEEE Trans. Antennas Propag., vol. 39, no. 6, pp. 754-757, Jun. 1991.

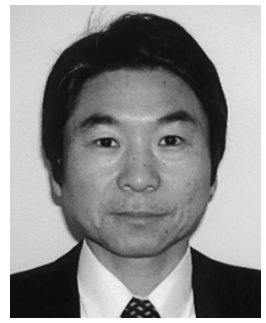

Hisamatsu Nakano (M'75-SM'87-F'92) received the B.E., M.E., and Dr.E. degrees in electrical engineering from Hosei University, Tokyo, Japan, in 1968,1970 , and 1974 , respectively.

Since 1973, he has been a member of the faculty of Hosei University, where he is now a Professor in the Electronic Informatics Department. He has published more than 190 refereed journal papers and 160 international symposium papers on antenna and relevant problems.

Dr. Nakano received the Best Paper Award from the IEE 5th International Conference on Antennas and Propagation in 1987 and the IEEE AP-S Best Application Paper Award (H. A. Wheeler Award) in 1994.

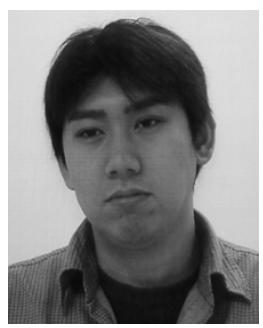

Kazuo Hitosugi received the B.E. degree in electronic informatics from Hosei University, Tokyo, Japan, in 2003, where he is currently working toward the M.E. degree.

Mr. Hitosugi is a Member of the Institute of Electronics, Information, and Communication Engineers (IEICE) of Japan.

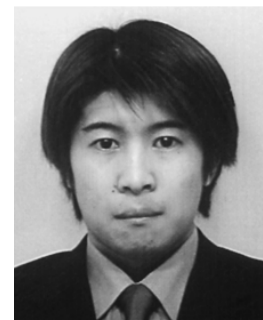

Naoki Tatsuzawa received the B.E. degree in electronic informatics from Hosei University, Tokyo, Japan, in 2003, where he is currently working toward the M.E. degree.

Mr. Tatsuzawa is a Member of the Institute of Electronics, Information, and Communication Engineers (IEICE) of Japan.

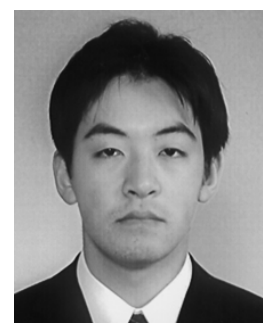

Daisuke Togashi received the B.E. degree in electronic informatics from Hosei University, Tokyo, Japan, in 2003, where he is currently working toward the M.E. degree.

Mr. Togashi is a Member of the Institute of Electronics, Information, and Communication Engineers (IEICE) of Japan.

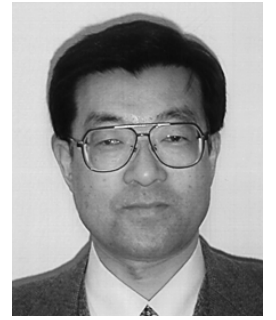

Hiroaki Mimaki (M'94) received the B.E. degree in electrical engineering from Tokyo Denki University, Tokyo, Japan, in 1976 and the M.E. degree in electrical engineering from Hosei University, Tokyo, Japan, in 1981.

He is currently an Assistant at Hosei University. His research interests are in thin wire antennas.

Mr. Mimaki is a Member of the Institute of Electronics, Information, and Communication Engineers (IEICE) of Japan.

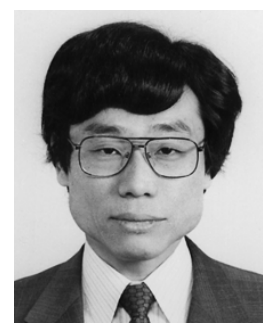

Junji Yamauchi (M'85) was born in Nagoya, Japan, on August 23, 1953. He received the B.E., M.E., an Dr.E. degrees from Hosei University, Tokyo, Japan, in 1976, 1978, and 1982, respectively.

From 1984 to 1988 , he served as a Lecturer in the Electrical Engineering Department of Tokyo Metropolitan Technical College. Since 1988, he has been a member of the faculty of Hosei University, where he is now a Professor of Electronic Informatics. His research interests include optical waveguides and circularly polarized antennas. He is the author of Propagating Beam Analysis of Optical waveguides (Hertfordshire, U.K.: Research Studies Press, 2003).

Dr. Yamauchi is a Member of the Optical Society of America (OSA), Washington, DC and the Institute of Electronics, Information and Communication Engineers (IEICE) of Japan. 